\title{
On Andrica's Conjecture, Cramér's Conjecture, gaps Between Primes and Jacobi Theta Functions I
}

\section{Prof. Dr. Raja Rama Gandhi and Edigles Guedes}

${ }^{1}$ Resource person in Math for Oxford University Press, Professor in Math, BITS-Vizag.

${ }^{2}$ World order Number Theorist, Pernambuco, Brazil.

ABSTRACT. The main objective of this paper is to develop upper and lower bound for the Andrica conjecture, gaps between primes, using Jacobi elliptic functions.

\section{INTRODUCTION}

In [1, p. 34] Richard K. Guy posted that Dorin Andrica conjectures that, for all natural $n$, we have

$$
\sqrt{p_{n+1}}-\sqrt{p_{n}}<1
$$

consequently, dividing both sides of the equation (1) by $\sqrt{p_{n}}$, we have

$$
\sqrt{\frac{p_{n+1}}{p_{n}}}-\frac{1}{\sqrt{p_{n}}}<1
$$

We will use the following notation for gaps between primes:

$$
g\left(p_{n}\right):=p_{n+1}-p_{n}
$$

that is related to Cramér's conjecture, which states

$$
\lim _{n \rightarrow \infty} \sup \frac{p_{n+1}-p_{n}}{\left(\log p_{n}\right)^{2}}=1,
$$

and the Rosser's theorem [2], which states that $p_{n}$ is $\operatorname{larger}$ than $n \log n$. This can be improved by the following pair of bounds:

$$
\log n+\log \log n-1<\frac{p_{n}}{n}<\log n+\log \log n
$$

for $n>6$.

\section{THEOREMS}

THEOREM 1. Let $k:=\frac{p_{n}}{p_{n+1}}$ to be a $k$ modulus and $n \geq 6$, then

$$
\left(\frac{\theta_{3}-\theta_{2}}{\theta_{2}}\right) \sqrt{n \log n+n \log \log n-n}<\sqrt{p_{n+1}}-\sqrt{p_{n}}<\left(\frac{\theta_{3}-\theta_{2}}{\theta_{2}}\right) \sqrt{n \log n+n \log \log n}
$$

where $\theta_{2}$ and $\theta_{3}$ are Jacobi theta functions.

Proof. Firstly, we consider the sequence of prime numbers

$$
2<3<5<7<11<13<17<19<\cdots p_{n-2}<p_{n-1}<p_{n}<p_{n+1} .
$$

Second, we note that

$$
0<\frac{2}{3}<1,0<\frac{3}{5}<1,0<\frac{5}{7}<1,0<\frac{7}{11}<1,0<\frac{11}{13}<1,0<\frac{13}{17}<1,0<\frac{17}{19}<1, \ldots
$$




$$
0<\frac{p_{n-2}}{p_{n-1}}<1,0<\frac{p_{n-1}}{p_{n}}<1,0<\frac{p_{n}}{p_{n+1}}<1
$$

Then, we define that

$$
k:=k_{n, n+1}=\frac{p_{n}}{p_{n+1}} \Leftrightarrow p_{n+1}=\frac{p_{n}}{k}
$$

where $k_{n, n+1}$ is the $k$ modulus.

Substituting (6) in the left-hand side of (1), we find

$$
\sqrt{p_{n+1}}-\sqrt{p_{n}}=\frac{\sqrt{p_{n}}}{k^{1 / 2}}-\sqrt{p_{n}}=\sqrt{p_{n}}\left(\frac{1-k^{1 / 2}}{k^{1 / 2}}\right) .
$$

In $[3$, p. 83$]$, we knew that

$$
k^{1 / 2}=\frac{\theta_{2}}{\theta_{3}}=\frac{\theta_{2}(0)}{\theta_{3}(0)}=\frac{\theta_{2}(0 \mid \tau)}{\theta_{3}(0 \mid \tau)}
$$

where $\tau$ is the parameter and $\theta_{2}(z \mid \tau)$ and $\theta_{3}(z \mid \tau)$ are Jacobi theta functions.

We set (8) in (7)

$$
\sqrt{p_{n+1}}-\sqrt{p_{n}}=\sqrt{p_{n}}\left(\frac{1-\frac{\theta_{2}}{\theta_{3}}}{\frac{\theta_{2}}{\theta_{3}}}\right)=\sqrt{p_{n}}\left(\frac{\frac{\theta_{3}-\theta_{2}}{\theta_{3}}}{\frac{\theta_{2}}{\theta_{3}}}\right)=\sqrt{p_{n}}\left(\frac{\theta_{3}-\theta_{2}}{\theta_{2}}\right) .
$$

From (3) and (9), we conclude that

$\left(\frac{\theta_{3}-\theta_{2}}{\theta_{2}}\right) \sqrt{n \log n+n \log \log n-n}<\sqrt{p_{n+1}}-\sqrt{p_{n}}<\left(\frac{\theta_{3}-\theta_{2}}{\theta_{2}}\right) \sqrt{n \log n+n \log \log n}$

COROLLARY 1. Let $k:=\frac{p_{n}}{p_{n+1}}$ to be a $k$ modulus, then Andrica's conjecture is equivalent to

$$
\sqrt{p_{n}}<\frac{\theta_{2}}{\theta_{3}-\theta_{2}}
$$

Proof. Dividing both members of (9) by $\sqrt{p_{n}}$, we have

$$
\sqrt{\frac{p_{n+1}}{p_{n}}}-\left(\frac{\theta_{3}-\theta_{2}}{\theta_{2}}\right)=1
$$

Comparing (2) with (10) and after some algebraic manipulation, we find

$$
\frac{\theta_{3}-\theta_{2}}{\theta_{2}}<\frac{1}{\sqrt{p_{n}}}
$$

therefrom,

$$
\sqrt{p_{n}}<\frac{\theta_{2}}{\theta_{3}-\theta_{2}}
$$

THEOREM 2. Let $k:=\frac{p_{n}}{p_{n+1}}$ to be a $k$ modulus, then

$$
\left(\frac{\theta_{3}^{2}-\theta_{2}^{2}}{\theta_{2}^{2}}\right)(n \log n+n \log \log n-n)<g\left(p_{n}\right)<\left(\frac{\theta_{3}^{2}-\theta_{2}^{2}}{\theta_{2}^{2}}\right)(n \log n+n \log \log n)
$$


where $\theta_{2}$ and $\theta_{3}$ are Jacobi theta functions.

Proof. We define that

$$
k:=k_{n, n+1}=\frac{p_{n}}{p_{n+1}} \Leftrightarrow p_{n+1}=\frac{p_{n}}{k}
$$

where $k_{n, n+1}$ is the $k$ modulus.

We consider that

$$
g\left(p_{n}\right):=p_{n+1}-p_{n}=\frac{p_{n}}{\left(k^{1 / 2}\right)^{2}}-p_{n}=p_{n}\left[\frac{1-\left(k^{1 / 2}\right)^{2}}{\left(k^{1 / 2}\right)^{2}}\right]
$$

In $[2$, p. 83$]$, we knew that

$$
k^{1 / 2}=\frac{\theta_{2}}{\theta_{3}}=\frac{\theta_{2}(0)}{\theta_{3}(0)}=\frac{\theta_{2}(0 \mid \tau)}{\theta_{3}(0 \mid \tau)^{\prime}}
$$

where $\tau$ is the parameter and $\theta_{2}(z \mid \tau)$ and $\theta_{3}(z \mid \tau)$ are Jacobi theta functions.

We set (14) in (13)

$$
g\left(p_{n}\right)=p_{n+1}-p_{n}=p_{n}\left(\frac{1-\frac{\theta_{2}^{2}}{\theta_{3}^{2}}}{\frac{\theta_{2}^{2}}{\theta_{3}^{2}}}\right)=p_{n}\left(\frac{\frac{\theta_{3}^{2}-\theta_{2}^{2}}{\theta_{3}^{2}}}{\frac{\theta_{2}}{\theta_{3}^{2}}}\right)=p_{n}\left(\frac{\theta_{3}^{2}-\theta_{2}^{2}}{\theta_{2}^{2}}\right)
$$

From (3) and (15), we conclude that

$$
\left(\frac{\theta_{3}^{2}-\theta_{2}^{2}}{\theta_{2}^{2}}\right)(n \log n+n \log \log n-n)<g\left(p_{n}\right)<\left(\frac{\theta_{3}^{2}-\theta_{2}^{2}}{\theta_{2}^{2}}\right)(n \log n+n \log \log n)
$$

THEOREM 3. Let $k:=\frac{p_{n}}{p_{n+1}}$ to be a $k$ modulus, then

$$
\left(\frac{\theta_{4}^{4}}{\theta_{2}^{4}}\right)(n \log n+n \log \log n-n)^{2}<p_{n+1}^{2}-p_{n}^{2}<\left(\frac{\theta_{4}^{4}}{\theta_{2}^{4}}\right)(n \log n+n \log \log n)^{2},
$$

where $\theta_{2}$ and $\theta_{3}$ are Jacobi theta functions.

Proof. We define that

$$
k:=k_{n, n+1}=\frac{p_{n}}{p_{n+1}} \Leftrightarrow p_{n+1}=\frac{p_{n}}{k}
$$

where $k_{n, n+1}$ is the $k$ modulus.

We consider that

$$
p_{n+1}^{2}-p_{n}^{2}=\frac{p_{n}^{2}}{\left(k^{1 / 2}\right)^{4}}-p_{n}^{2}=p_{n}^{2}\left[\frac{1-\left(k^{1 / 2}\right)^{4}}{\left(k^{1 / 2}\right)^{4}}\right]
$$

In $[2$, p. 83], we knew that

$$
k^{1 / 2}=\frac{\theta_{2}}{\theta_{3}}=\frac{\theta_{2}(0)}{\theta_{3}(0)}=\frac{\theta_{2}(0 \mid \tau)}{\theta_{3}(0 \mid \tau)^{\prime}}
$$

where $\tau$ is the parameter and $\theta_{2}(z \mid \tau)$ and $\theta_{3}(z \mid \tau)$ are Jacobi theta functions. 
We set (18) in (17)

$$
p_{n+1}^{2}-p_{n}^{2}=p_{n}^{2}\left(\frac{1-\frac{\theta_{2}^{4}}{\theta_{3}^{4}}}{\frac{\theta_{2}^{4}}{\theta_{3}^{4}}}\right)=p_{n}^{2}\left(\frac{\frac{\theta_{3}^{4}-\theta_{2}^{4}}{\theta_{3}^{4}}}{\frac{\theta_{2}^{4}}{\theta_{3}^{4}}}\right)=p_{n}^{2}\left(\frac{\theta_{3}^{4}-\theta_{2}^{4}}{\theta_{2}^{4}}\right)=p_{n}^{2}\left(\frac{\theta_{4}^{4}}{\theta_{2}^{4}}\right)
$$

to see [3, p. 84] which states $\theta_{2}^{4}+\theta_{4}^{4}=\theta_{3}^{4}$, the Jacobi identity.

From (3) and (19), we conclude that

$$
\left(\frac{\theta_{4}^{4}}{\theta_{2}^{4}}\right)(n \log n+n \log \log n-n)^{2}<p_{n+1}^{2}-p_{n}^{2}<\left(\frac{\theta_{4}^{4}}{\theta_{2}^{4}}\right)(n \log n+n \log \log n)^{2} . \square
$$

\section{ACKNOWLEDGMENTS}

I thank Prof. Dr. K. Raja Rama Gandhi for their encouragement and support during the development of this paper.

\section{REFERENCES}

[1] Guy, Richard K., Unsolved Problems in Number Theory, Springer, 2000.

[2] http://en.wikipedia.org/wiki/Prime_number_theorem, avaliable in April 22, 2013.

[3] Armitage, J. V. and Eberlein, W. F., Elliptic Functions, London Mathematical Society, 2006. 